\title{
Regorafenib (Stivarga) pharmacologically targets epithelial- mesenchymal transition in colorectal cancer
}

\author{
Li-Ching Fan ${ }^{1,2}$, Hao-Wei Teng ${ }^{3,7}$, Chung-Wai Shiau ${ }^{4}$, Wei-Tien Tai ${ }^{1,2}$, Man-Hsin \\ Hung $^{5,7}$, Shung-Haur Yang ${ }^{6,7}$, Jeng-Kai Jiang ${ }^{6,7}$, Kuen-Feng Chen ${ }^{1,2}$ \\ ${ }^{1}$ Department of Medical Research, National Taiwan University Hospital, Taipei, Taiwan \\ ${ }^{2}$ National Center of Excellence for Clinical Trial and Research, National Taiwan University Hospital, Taipei, Taiwan \\ ${ }^{3}$ Division of Hematology and Oncology, Department of Medicine, Taipei Veterans General Hospital, Taipei, Taiwan \\ ${ }^{4}$ Institute of Biopharmaceutical Sciences, National Yang-Ming University, Taipei, Taiwan \\ ${ }^{5}$ Division of Medical Oncology, Department of Oncology, Taipei Veterans General Hospital, Taipei, Taiwan \\ ${ }^{6}$ Division of Colon and Rectal Surgery, Department of Surgery, Taipei Veterans General Hospital, Taipei, Taiwan \\ ${ }^{7}$ School of Medicine, National Yang-Ming University, Taipei, Taiwan \\ Correspondence to: Kuen-Feng Chen, email: kfchen1970@ntu.edu.tw \\ Keywords: regorafenib, EMT, SHP-1, STAT3, CRC \\ Received: March 11,2016 Accepted: August 13, $2016 \quad$ Published: August 26, 2016
}

\section{ABSTRACT}

Epithelial-to-mesenchymal transition (EMT) is well-known to evoke cancer invasion/metastasis, leading to a high frequency of mortality in patients with metastatic colorectal cancer (mCRC). Protein tyrosine phosphatase (PTPase)-targeted therapy has been identified as a novel cancer therapeutic. Previously, we proved that sorafenib with anti-EMT potency prevents TGF- $\beta 1$-induced EMT/invasion by directly activating SH2-domain-containing phosphatase 1 (SHP-1)-dependent p-STAT3 ${ }^{\text {Tyr705 }}$ suppression in hepatocellular carcinoma. Regorafenib has a closely related chemical structure as sorafenib and is approved for the pharmacotherapy of mCRC. Herein, we evaluate whether regorafenib activates PTPase SHP-1 in the same way as sorafenib to abolish EMT-related invasion/metastasis in CRC. Notably, regorafenib exerted potent anti-EMT activity to curb TGF- $\beta 1$-induced EMT/invasion in vitro as well inhibited lung metastatic outgrowth of SW480 mesenchymal cells in vivo. Mechanistically, regorafenib-enhanced SHP-1 activity significantly impeded TGF- $\beta 1$-induced EMT/ invasion via low p-STAT3 ${ }^{\text {Tyr705 }}$ level as proved by a SHP-1 inhibitor or siRNA-mediated SHP-1 depletion. Conversely, overexpression of SHP-1 further enhanced the inhibitory effects of regorafenib on TGF- $\beta 1$-induced p-STAT3 ${ }^{\text {Tyr705 }}$ and EMT/invasion. Regorafenib directly activates SHP-1 by potently relieving the autoinhibited $\mathrm{N}-\mathrm{SH} 2$ domain of SHP-1 to inhibit TGF- $\beta 1$-induced $\mathrm{p}-\mathrm{STAT}^{\mathrm{T}}{ }^{\mathrm{Tr} 705}$ and EMT/invasion. Importantly, the clinical evidence indicated that SHP-1 was positively correlated with E-cadherin and that significantly determined the overall survival of CRC patients. This result further confirms our in vitro data that SHP-1 is a negative regulatory PTPase in EMT regulation and serves as a pharmacological target for mCRC therapy. Collectively, activating PTPase SHP-1 by regorafenib focusing on its anti-EMT activity might be a useful pharmacotherapy for $\mathrm{mCRC}$.

\section{INTRODUCTION}

Metastatic colorectal cancer (mCRC) is a significant cause of mortality and morbidity worldwide because of its high biological heterogeneity. Despite the promising outcome of targeted therapies for $\mathrm{mCRC}$, including those using anti-EGFR antibodies, and VEGF inhibitors, the drug resistance to the primary and secondary therapy remains a clinical challenge in mCRC. Most notably, regorafenib (BAY 73-4506, commercial name Stivarga) was approved by the FDA in September 2012 and is used for mCRC patients as a last-line therapy. It is an oral multitargeted 
tyrosine kinase inhibitor (TKI) of BRAF, VEGFR-1, -2, -3, KIT, TIE-2, PDGFR- $\beta$, FGFR-1, RET and RAF- 1 that is particularly involved in oncogenesis and angiogenesis [1, 2]. Evidence from the murine metastatic CRC model also indicates that regorafenib potently abolishes angiogenesis and metastasis in advanced CRCs [3]. Importantly, epithelial-to-mesenchymal transition (EMT) is well known to be associated with tumor invasion and metastasis, including mCRC [4-7]. Whether regorafenib exerts antimetastatic activity is because of its capability to inhibit EMT in CRC is not fully understood and yet to be characterized.

Src homology region 2 (SH2) domain-containing phosphatase 1 (SHP-1), a non-receptor protein tyrosine phosphatase (PTP), has been identified as a key negative regulator of cytokine signaling and immune cell activation $[8,9]$. Interestingly, it also shows significant preclinical antitumor activity by triggering apoptosis in vitro and suppressing tumor formation in vivo via the PTP activity of SHP-1 that negatively targets p-STAT3 ${ }^{\text {Tyr705 }}$ signals in various cancer types [10-13]. Of note, STAT3 signaling is not only important for cancer cells in antagonizing apoptosis but has also been reported to be associated with cancer EMT by STAT3 transcriptional activation of EMT inducers TWIST1 and ZEB1 involved in E-cadherin repression [1417]. The available clinical evidence of p-STAT3 $3^{\text {Tyr705 }}$ in EMT progression also reveals a significant positive correlation with tumor, lymph node and metastasis stages (TNM) [17, 18]. Most notably, our team previously discovered that SHP1 acts as a strong suppressor, preventing TGF- $\beta 1$-induced EMT characteristics in vitro and inhibiting metastatic growth in vivo in hepatocellular carcinoma (HCC) by exerting tyrosine phosphatase activity that downregulates p-STAT3 ${ }^{\text {Tyr705 }}$ directly [14]. Moreover, sorafenib, a targeted therapeutic drug for HCC, has been identified as a SHP-1 agonist that increases SHP-1 activity directly through its docking potential to N-SH2 and the catalytic PTP domain of SHP-1, leading to the relief of autoinhibition of SHP-1 $[14,19]$. This contributes to the depletion of p-STAT3 ${ }^{\text {Tyr705 }}$ mediated EMT characteristics induced by TGF- $\beta 1$ [14]. Our findings therefore present a novel relationship between SHP-1 and HCC EMT and reveal that targeting of the SHP1-STAT3 axis by sorafenib may be an attractive strategy for HCC patients with metastasis.

Regorafenib (Fluror-sorafenib) has a chemical structure that is closely related to sorafenib and is the first approved pharmacotherapy for mCRC patients who have exhausted current standard therapies. In this study, we clarify whether regorafenib potently inhibits CRC EMT and metastasis in the same way as sorafenib by directly targeting the SHP-1-STAT3 axis. The identification of SHP-1 as a suppressor of CRC EMT and a druggable target of regorafenib focusing on its anti-EMT activity will advance our knowledge regarding the novel relationship between regorafenib and anti-EMT potency and highlights a therapeutic strategy for $\mathrm{mCRC}$ centering on the negative regulatory PTPase SHP-1.

\section{RESULTS}

\section{A novel link between the SHP-1-E-cadherin axis and overall survival of CRC patients}

First, we used immunohistochemistry (IHC) to examine the expression level of SHP-1 and E-cadherin in $\mathrm{CRC}$ tissues and then investigated the clinical associations between SHP-1, E-cadherin and the clinicopathological parameters in CRC patients. $243 \mathrm{CRC}$ patients were enrolled for this clinical analysis. As shown in Table 1, neither SHP-1 nor E-cadherin expression had a significant correlation with gender, AJCC stage, pathology or lymphovascular emboli. Moreover, there was no correlation between SHP-1 expression and histological grading. However, a significant inverse correlation was observed between E-cadherin and histological grading ( $P$ $=0.034)$, revealing that poorly differentiated human CRC with high histological grading showed loss of E-cadherin at cell boundaries, further suggesting the occurrence of EMT/metastasis. Most notably, we observed that a significant positive correlation existed between SHP-1 and E-cadherin expression $(P=0.038)$ (Table 1$)$. In line with the results of statistical analysis, IHC patterns shown in CRC patient\#1 with strong SHP-1 expression had strong E-cadherin expression. In contrast, patient\#2 with weak SHP-1 expression had weak E-cadherin expression (Figure 1A). The overall survival (OS) curve performed by Kaplan-Meier analysis showed that no significant correlation between the median OS and SHP-1 expression $(P=0.187)$ (Supplementary Figure 1). Notably, the median OS was not available in patients with strong expression both in SHP-1 and E-cadherin (Figure 1B). However, patients with weak expression of both SHP-1 and E-cadherin had a more significant reduction in median OS than others $(P=0.004)$. These results suggest that SHP-1 is not an independent prognostic marker of CRC patients in our current study. Of note, patients with an elevated SHP1-E-cadherin axis display longer survival. Taken together, targeting SHP-1-E-cadherin axis may be an effective treatment for suppressing EMT-related metastasis in CRC.

\section{Loss of p-STAT3 ${ }^{\text {Tyr705 }}$ expression is associated with regorafenib-suppressed mesenchymal phenotype}

Regorafenib is a multitargeted tyrosine kinase (TKI) and the first approved treatment for metastatic CRC [2, $3]$. Recently, we reported that sorafenib can significantly inhibit TGF- $\beta 1$-induced EMT and invasion by SHP-1dependent STAT3 inhibition [14]. Next, we examined whether regorafenib exerts anti-EMT effects by the loss of p-STAT3 ${ }^{\text {Tyr705 }}$ expression. As shown in Figure 2A, dose-escalation of regorafenib-treated SW480 cells with mesenchymal characteristics showed a gradual decrease in invasive capability along with the decreased expression 
Table 1: The associations between SHP-1, E-cadherin and clinicopathological parameters were analyzed in 243 CRC patients

\begin{tabular}{|c|c|c|c|c|c|c|c|c|c|c|c|}
\hline \multirow{2}{*}{\multicolumn{2}{|c|}{ Clinicopathological parameters }} & \multicolumn{5}{|c|}{ SHP-1 } & \multicolumn{4}{|c|}{ E-cadherin } & \multirow[b]{3}{*}{$P$} \\
\hline & & \multicolumn{2}{|c|}{ Weak } & \multicolumn{2}{|c|}{ Strong } & \multirow[b]{2}{*}{$P$} & \multicolumn{2}{|c|}{ Weak } & \multicolumn{2}{|c|}{ Strong } & \\
\hline & & $\mathbf{n}$ & $\%$ & $\mathrm{n}$ & $\%$ & & $\mathrm{n}$ & $\%$ & $\mathbf{n}$ & $\%$ & \\
\hline \multirow[t]{2}{*}{ Gender } & Female & 40 & 36.0 & 44 & 33.6 & 0.690 & 45 & 33.8 & 39 & 35.8 & 0.752 \\
\hline & Male & 71 & 64.0 & 87 & 66.4 & & 88 & 66.2 & 70 & 64.2 & \\
\hline \multirow[t]{2}{*}{ Location } & Left & 57 & 51.4 & 85 & 64.9 & 0.033 & 77 & 57.9 & 65 & 59.6 & 0.785 \\
\hline & Right & 54 & 48.6 & 46 & 35.1 & & 56 & 42.1 & 44 & 40.4 & \\
\hline \multirow[t]{4}{*}{ AJCC Stage } & I & 6 & 5.4 & 11 & 8.4 & 0.309 & 7 & 5.3 & 10 & 9.2 & 0.127 \\
\hline & II & 36 & 32.4 & 31 & 23.7 & & 35 & 26.3 & 32 & 29.4 & \\
\hline & III & 29 & 26.1 & 44 & 33.6 & & 36 & 27.1 & 37 & 33.9 & \\
\hline & IV & 40 & 36.0 & 45 & 34.4 & & 55 & 41.4 & 30 & 27.5 & \\
\hline \multirow[t]{4}{*}{ Pathology } & Adenocarcinoma & 102 & 91.9 & 129 & 98.5 & 0.097 & 124 & 93.2 & 107 & 98.2 & 0.298 \\
\hline & Carcinoma & 1 & .9 & 0 & .0 & & 1 & .8 & 0 & .0 & \\
\hline & $\begin{array}{c}\text { Mucinous } \\
\text { adenocarcinoma }\end{array}$ & 7 & 6.3 & 2 & 1.5 & & 7 & 5.3 & 2 & 1.8 & \\
\hline & $\begin{array}{l}\text { Signet ring cell } \\
\text { carcinoma }\end{array}$ & 1 & .9 & 0 & .0 & & 1 & .8 & 0 & .0 & \\
\hline \multirow[t]{2}{*}{$\mathrm{T} 4$} & Not & 60 & 65.9 & 73 & 67.0 & 0.877 & 65 & 60.7 & 68 & 73.1 & 0.064 \\
\hline & Yes & 31 & 34.1 & 36 & 33.0 & & 42 & 39.3 & 25 & 26.9 & \\
\hline \multirow[t]{2}{*}{ Grade } & Low $(\mathrm{G} 1, \mathrm{G} 2)$ & 95 & 88.8 & 120 & 93.0 & 0.255 & 112 & 87.5 & 103 & 95.4 & 0.034 \\
\hline & $\operatorname{High}(\mathrm{G} 3, \mathrm{G} 4)$ & 12 & 11.2 & 9 & 7.0 & & 16 & 12.5 & 5 & 4.6 & \\
\hline \multirow[t]{2}{*}{$\begin{array}{l}\text { Lymphovascular } \\
\text { emboli }\end{array}$} & No & 80 & 76.9 & 94 & 75.2 & 0.761 & 93 & 75.0 & 81 & 77.1 & 0.705 \\
\hline & Yes & 24 & 23.1 & 31 & 24.8 & & 31 & 25.0 & 24 & 22.9 & \\
\hline \multirow[t]{2}{*}{ SHP-1 } & Weak & 111 & 100.0 & 0 & .0 & - & 69 & 51.9 & 42 & 38.5 & 0.038 \\
\hline & Strong & 0 & .0 & 131 & 100.0 & & 64 & 48.1 & 67 & 61.5 & \\
\hline \multirow[t]{2}{*}{ E-cadherin } & Weak & 69 & 62.2 & 64 & 48.9 & 0.038 & 133 & 100.0 & 0 & .0 & - \\
\hline & Strong & 42 & 37.8 & 67 & 51.1 & & 0 & .0 & 109 & 100.0 & \\
\hline
\end{tabular}

A

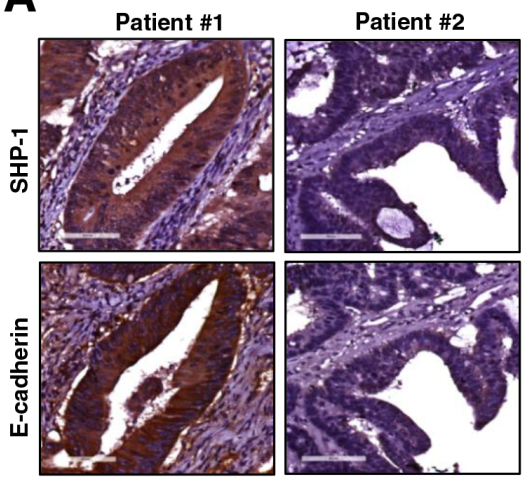

B

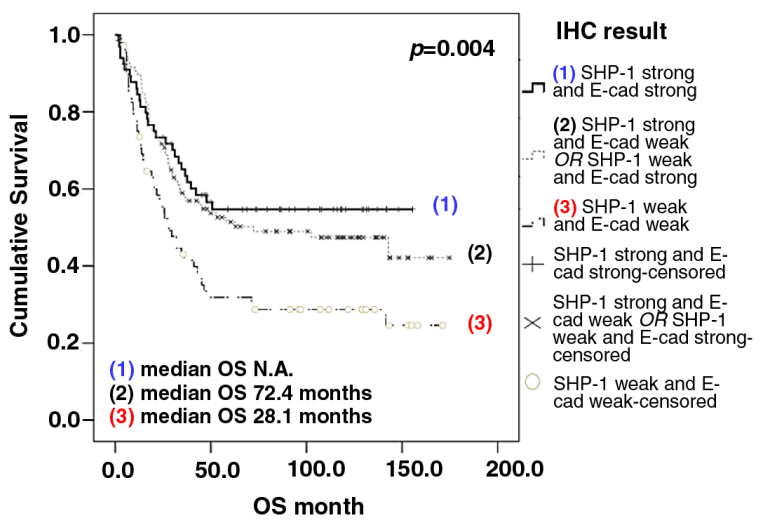

Figure 1: The expression of SHP-1 and E-cadherin correlates with overall survival of CRC patients. A. Representative immunohistochemical patterns revealed that clinical CRC patient\#1 with strong positive expression of SHP-1 had strong positive expression of E-cadherin. Patient\#2 with weak positive expression of SHP-1 had weak positive expression of E-cadherin. B. Patients with weak expression of both SHP-1 and E-cadherin had a more significant reduction in median OS than others. (N.A., not available) 
A
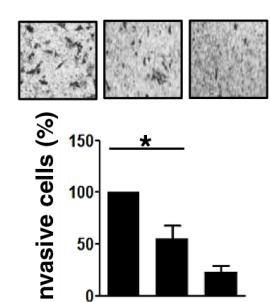

Regorafenib ( $\mu \mathrm{M}) \quad 0 \quad 2.5 \quad 5$

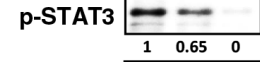

STAT3

Vimentin

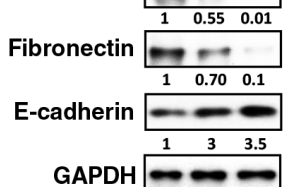

B
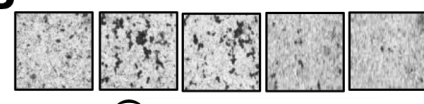

$\overbrace{}^{\circ} 250 * *$

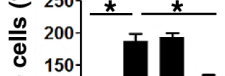

8150

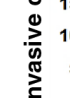

50

TGF- $\beta 1$

Regorafenib $(\mu \mathrm{M})$ - $\quad-12.55$

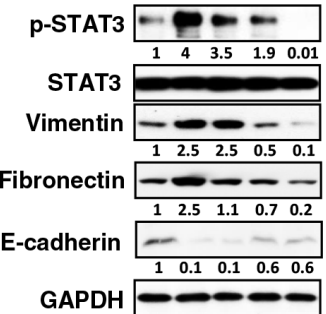

C

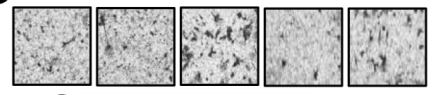

$\overbrace{}^{\circ} 250+*$ *

잉 200

$\sum_{\substack{0 \\ 0}}^{\infty} 100$

$\sum_{2}^{2} 50$

TGF- $\beta 1$ - -++

Regorafenib - + - +

STAT3 - - - -+

p-STAT3 $=-8 \quad$ ?

STAT

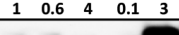

Vimentin \begin{tabular}{|llllll}
\hline & - & $\cdots$ & - & & - \\
\hline
\end{tabular}

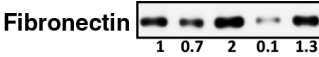

E-cadherin

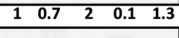

GAPDH

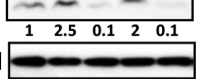

D
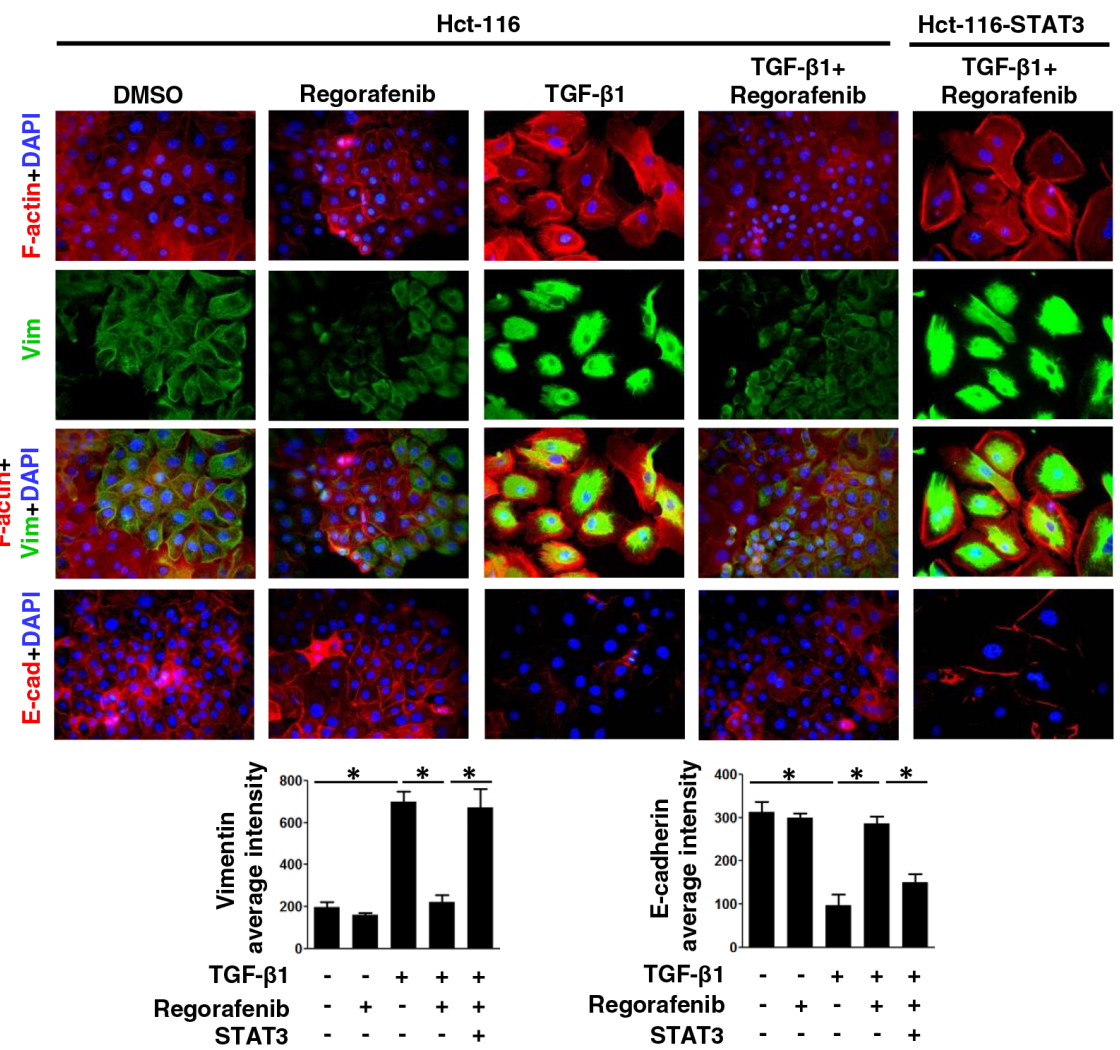

Figure 2: The inhibition of mesenchymal characteristics by regorafenib is dependent on p-STAT3Tyr705 downregulation. A. Western blotting of p-STAT3 ${ }^{\text {Tyr705 }}$, STAT3, mesenchymal (vimentin and fibronectin) and epithelial (E-cadherin) markers in SW480 cells $24 \mathrm{~h}$ after treatment with dose-escalation of regorafenib. GAPDH was used as a loading control. The invasive capability of cells was measured. The results are shown as mean $\pm \mathrm{SD}$ of three independent experiments $\left({ }^{*}, P<0.05\right)$. Data are presented as a percentage relative to control SW480 cells. B. Western blotting was used to detect the expression of p-STAT3 ${ }^{\text {Tyr705 }}$, STAT3, vimentin, fibronectin and E-cadherin in Hct-116 cells $24 \mathrm{~h}$ after either co-treatment with TGF- $\beta 1(10 \mathrm{ng} / \mathrm{ml})$ and/or the indicated dose of regorafenib $(1,2.5,5 \mu \mathrm{M})$. GAPDH was used as a loading control. Invasion assay was performed in cells. The results are shown as mean $\pm \mathrm{SD}$ of three independent experiments $(*, P<0.05)$. Data are presented as the percentage relative to control Het-116 cells. C. Western blotting of p-STAT3 ${ }^{\text {Tyros }}$, STAT3, vimentin, fibronectin and E-cadherin in STAT3-overexpressing Hct-116 cells $24 \mathrm{~h}$ after either co-treatment with TGF- $\beta 1$ (10 ng/ $\mathrm{ml})$ and/or regorafenib $(2.5 \mu \mathrm{M})$. GAPDH was used as a loading control. Invasion assay was performed in these cells. The results are shown as mean $\pm \mathrm{SD}$ of three independent experiments $(*, P<0.05)$. Data are presented as the percentage relative to control Hct-116 cells. D. Immunofluorescence microscopy analysis of rhodamine phalloidin-stained F-actin, DAPI-stained nuclei, vimentin and E-cadherin in the cells shown in (C). Image analysis of vimentin and E-cadherin was quantified by two independent experiments. $(*, P<0.05)$ 
of p-STAT3 $3^{\text {Tyr705 }}$ and mesenchymal markers (vimentin and fibronectin) but had an increased expression of E-cadherin, an epithelial marker. Moreover, when dose-escalation of regorafenib was used to treat epithelial-like cells Hct-116, it resulted in an increase of E-cadherin expression inhibited by TGF- $\beta 1$. However, it caused significant abolishment of invasive capability as well as decreasing the levels of p-STAT3 ${ }^{\text {Tyr705 }}$ and mesenchymal markers (vimentin and fibronectin) that were increased by TGF- $\beta 1$ (Figure 2B). The morphological characteristics of TGF- $\beta 1$-treated Hct116 cells showed the obvious lost cell-cell contacts and displayed dramatic extensively flattened and elongated leading-trailing mesenchymal morphology along with the increased expression of vimentin and F-actin but concomitant inhibition of E-cadherin expression (Figure 2D). Most notably, these effects were further antagonized by regorafenib and resulted in the acquisition of epitheliallike features, including F-actin-stained cell-cell contacts and increased E-cadherin expression, but reduced the level of vimentin (Figure 2D). These results therefore suggest that the pharmacological mechanism by which regorafenib abolishes the mesenchymal characteristics of CRC cells is likely through the loss of p-STAT3 ${ }^{\text {Tyr705. }}$. We therefore investigated whether STAT3 overexpression rescues the epithelial phenotype in Hct-116 cells cotreated with TGF- $\beta 1$ and regorafenib and finally enables the occurrence of EMT. Importantly, overexpression of STAT3 in TGF- $\beta 1$ and regorafenib co-treated Hct-116 cells not only enhanced the expression of p-STAT3 ${ }^{\text {Tyr705 }}$ and mesenchymal markers (vimentin and fibronectin) but also promoted the invasion and exhibited the mesenchymal morphologic characteristics including higher vimentin but lower E-cadherin expression, compared with Hct-116 cells co-treated with TGF- $\beta 1$ and regorafenib (Figure $2 \mathrm{C}$ and 2D). Our data, therefore, provide evidence to identify that regorafenib serves as an anti-EMT therapeutic drug in CRC by antagonizing p-STAT3 ${ }^{\text {Tyr705 }}$ signaling.

\section{Regorafenib-enhanced SHP-1 tyrosine phosphatase activity is required for the blockage of EMT through p-STAT3 ${ }^{\text {Tyr705 }}$ downregulation}

Because SHP-1 tyrosine phosphatase activity could negatively regulate HCC EMT by mediating the inactivation of STAT3 [14], we then evaluated whether SHP-1 tyrosine phosphatase activity is involved in the effects of regorafenib on reducing TGF- $\beta 1$-induced p-STAT3 ${ }^{\mathrm{Tyr} 705}$, which is required for EMT. We found that regorafenib significantly increased SHP-1 activity in SW480 cells in a dose-dependent manner (Figure 3A, upper panels). Regorafenib-enhanced SHP-1 activity was also seen in Hct-116 cells co-treated with TGF- $\beta 1$ and dose-escalation of regorafenib, compared with TGF- $\beta 1$-treated cells (Figure 3A, lower panels). Next, in order to know whether regorafenib reduced TGF- $\beta 1$ induced p-STAT3 ${ }^{\text {Tyr705 }}$ expression is SHP-1-dependent, we employed siRNA to deplete SHP-1 and found that knockdown of SHP-1 in TGF- $\beta 1$-treated Hct-116 cells significantly increased both the levels of p-STAT3 ${ }^{\text {Tyr705 }}$ and mesenchymal markers as well promoting invasive capability, compared with TGF- $\beta 1$-treated cancer cells (Figure 3B, left panels). However, their expression levels were not further altered in SHP-1-depleted cancer cells co-treated with TGF- $\beta 1$ and regorafenib (Figure 3B, left panels). These observations were further confirmed by using SHP-1 phosphatase-specific inhibitor (PTPIII) to inhibit SHP-1 activity as shown in Figure 3B, right panels. These findings indicate that SHP-1 plays a key role in mediating the effect of regorafenib on reducing TGF- $\beta 1$-induced $p-S T A T 3^{\text {Tyr705 }}$ activation. In contrast, overexpression of SHP-1 significantly reduced the effect of TGF- $\beta 1$ on the levels of p-STAT3 ${ }^{\text {Tyr705 }}$ and mesenchymal markers in Hct-116 and HT-29 cells and the treatment of regorafenib further enhanced these effects by SHP-1 overexpression (Figure 3C). Most importantly, regorafenib upregulated the activity of SHP-1 significantly in SHP-1-overexpressed Hct-116 and HT-29 cells treated with TGF- $\beta 1$ (Figure 3D). Taken together, these results clearly demonstrate that regorafenib abolished the effects of TGF- $\beta 1$ on p-STAT3 ${ }^{\text {Tyr705 }}$ and EMT was through the enhancement of the SHP-1 activity, resulting in downregulation of $\mathrm{p}-\mathrm{STAT} 3^{\mathrm{Ty} 705}$ (Figure $3 \mathrm{E}$ ).

\section{Regorafenib directly activates SHP-1 by potently relieving the autoinhibited $\mathrm{N}-\mathrm{SH} 2$ domain of SHP-1 to inhibit p-STAT3 ${ }^{\text {Tyr705 }}$ level and EMT}

To further investigate the druggable mechanism through which regorafenib negatively regulates EMT by increasing SHP-1 tyrosine phosphatase activity, we transfected mesenchymal cells SW480 with wildtype or mutant SHP-1 (D61A and C453S). In SHP-1, intramolecular inhibition occurs through association of the N-SH2 domain with the PTP catalytic domain which is stabilized by a salt bridge between Asp61 (D61) and Lys362 (Figure 4A, upper panels). The D61A point mutant mimics the open conformation of SHP-1 and serves as a constitutive activator. $\mathrm{C} 453 \mathrm{~S}$ is the catalytic-dead mutant of SHP-1 (Figure 4A, upper panels). The D61A mutant SHP-1 showed an increase in SHP-1 tyrosine phosphatase activity (Figure 4A, lower panels) but displayed a marked decrease in p-STAT3 ${ }^{\text {Tyr705 }}$ and mesenchymal markers vimentin and fibronectin, compared with wild-type and C453S mutant SHP-1 (Figure 4B, left panels). Importantly, regorafenib significantly increased SHP-1 tyrosine phosphatase activity in wild-type SHP-1-transfected but not in D61A or C453S mutant SHP-1-transfected SW480 cells (Figure 4A, lower panels), suggesting that regorafenib increases SHP-1 tyrosine phosphatase activity through the direct disruption of the interaction between the N-SH2 domain and the PTP catalytic domain of SHP-1 that further relieves the autoinhibition of SHP-1. 
A

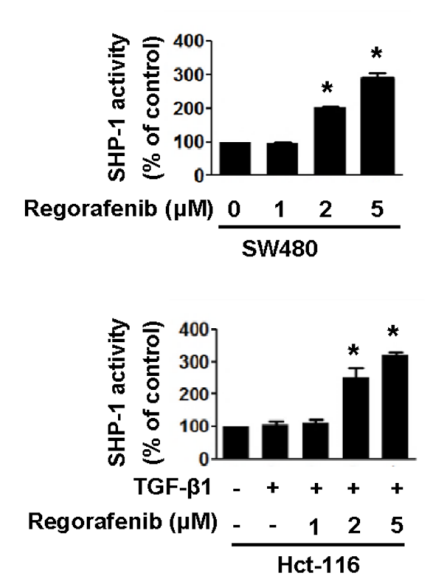

C

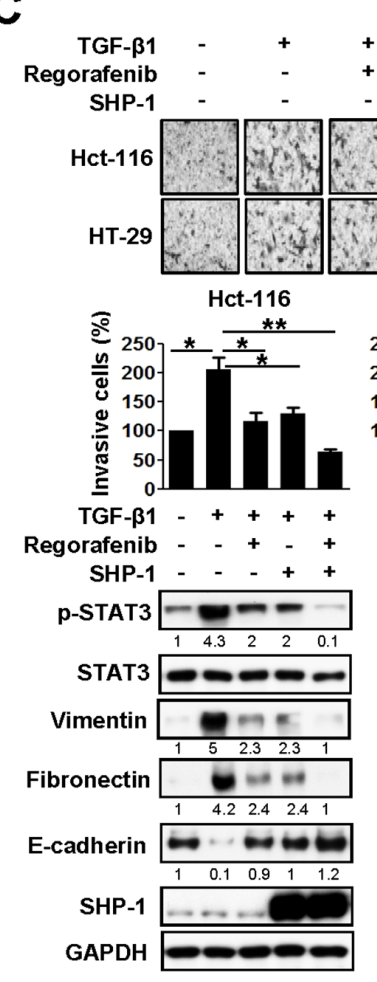

B

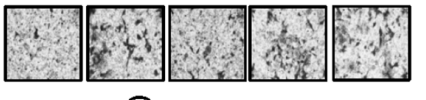

ङ

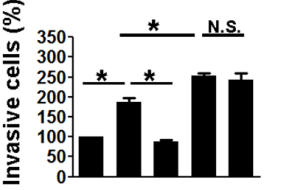

TGF- $\beta 1-+++$

Regorafenib - - + - +

siSHP-1

p-STAT3

STAT3

Vimentin

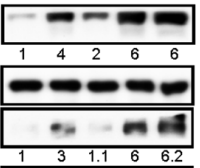

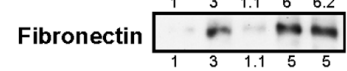

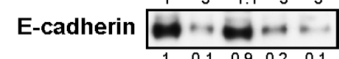

SHP-1 $\cdots \cdots$

GAPDH

D
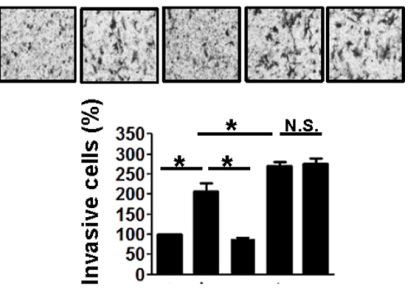

TGF-B1 - + + +

Regorafenib - - + - +

SHP-1 inhibitor - - - + +

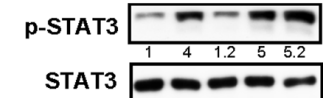

Vimentin

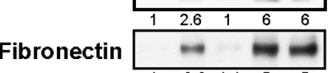

\begin{tabular}{|llllll}
\hline 1 & 3.3 & 1.1 & 5 & 5 \\
\hline
\end{tabular}

E-cadherin $\ldots \ldots \ldots$

GAPDH
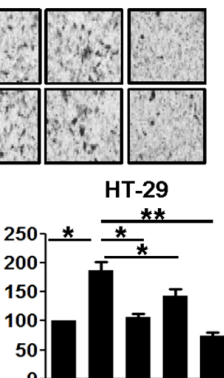

$\begin{array}{lllll}- & + & + & + & + \\ - & - & + & - & +\end{array}$
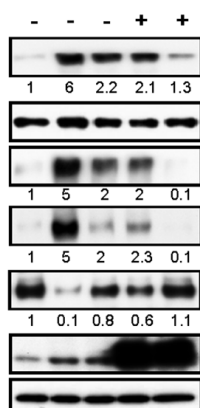

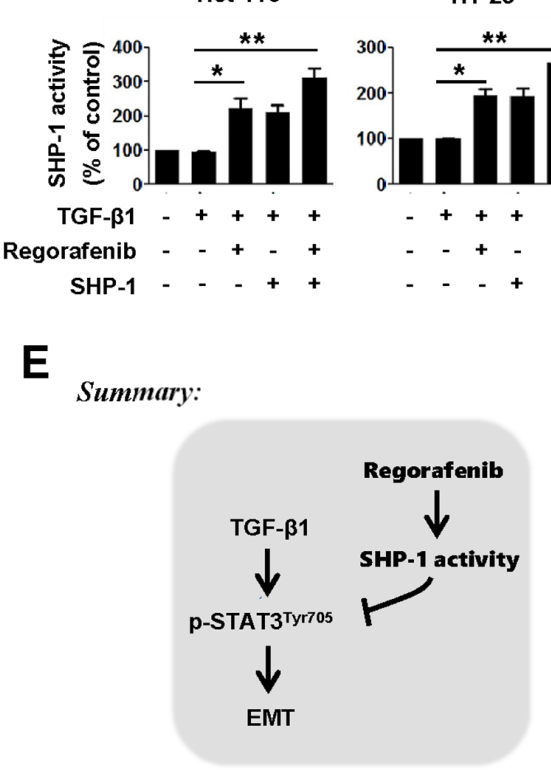

Figure 3: Abolishment of the effects of TGF- $\beta 1$ on p-STAT3Tyr705 and EMT by regorafenib is through the enhancement of SHP-1 activity, resulting in down-regulation of p-STAT3Tyr705. A. Upper panels, SHP-1 activity was measured in SW480 cells treated with dose-escalation of regorafenib for $24 \mathrm{~h}$. Lower panels, SHP-1 activity was measured in the Hct-116 cells either co-treated with TGF- $\beta 1(10 \mathrm{ng} / \mathrm{ml})$ and/or the indicated dose of regorafenib for $24 \mathrm{~h}$. The results are shown as mean $\pm \mathrm{SD}$ of three independent experiments $(*, P<0.05)$. Data are presented as the percentage relative to control SW480 or Hct-116 cells. B. Left panels, western blotting of p-STAT3 ${ }^{\text {Tyr705}}$, STAT3, vimentin, fibronectin and E-cadherin in TGF- $\beta 1$-treated Hct-116 cells $24 \mathrm{~h}$ after either co-treatment with or without SHP-1 siRNA $(25 \mathrm{nM})$ and/or regorafenib $(2.5 \mu \mathrm{M})$. Right panels, western blotting of p-STAT3 ${ }^{\text {Tyr705}}$, STAT3, vimentin, fibronectin and E-cadherin in TGF- $\beta 1$-treated Hct-116 cells $24 \mathrm{~h}$ after either co-treatment with or without SHP-1 inhibitor $(20 \mathrm{nM})$ and/or regorafenib $(2.5 \mu \mathrm{M})$. GAPDH was used as a loading control. Invasion assay was performed in these cells shown in left and right panels. The results are shown as mean $\pm \mathrm{SD}$ of three independent experiments $(*, P<0.05$; N.S., non-significant). Data are presented as the percentage relative to

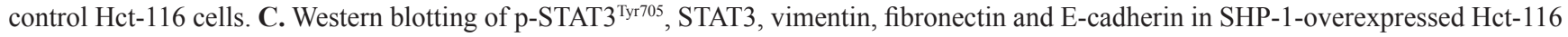
and HT-29 cells $24 \mathrm{~h}$ after either co-treatment with TGF- $\beta 1(10 \mathrm{ng} / \mathrm{ml})$ and/or regorafenib $(2.5 \mu \mathrm{M})$. GAPDH was used as a loading control. The invasive capability was measured in these cells. The results are shown as mean $\pm \mathrm{SD}$ of three independent experiments $\left(^{*}, P<0.05\right.$, **, $P<0.01$ ). Data are presented as a percentage relative to the control Hct-116 or HT-29 cells. D. SHP-1 activity was performed in cells shown in (C). The results are shown as mean $\pm \mathrm{SD}$ of three independent experiments $(*, P<0.05, * *, P<0.01)$. Data are presented as a percentage relative to control Hct-116 or HT-29 cells. E. A mechanistic representation showing that regorafenib prevents TGF- $\beta 1$-induced EMT mediated through regorafneib-enhanced SHP-1 activity that effectively downregulates p-STAT3 ${ }^{\text {Tyr705 }}$ expression. 
In addition, wild-type SHP-1-transfected SW480 cells showed a marked decrease in expression of p-STAT3 ${ }^{\text {Tyr705 }}$ and mesenchymal markers (vimentin and fibronectin) but had a significant increase expression of E-cadherin after regorafenib treatment (Figure 4B, left panels). These cells displayed a significant reduction in invasive capability
(Figure 4B, right panels). However, this observation was not seen in D61A or C453S mutant SHP-1-transfected cells following treatment with regorafenib (Figure 4B). Our data therefore identifies SHP-1 as a druggable target of regorafenib verified by the evidence that regorafenib pharmacologically increases SHP-1 activity through
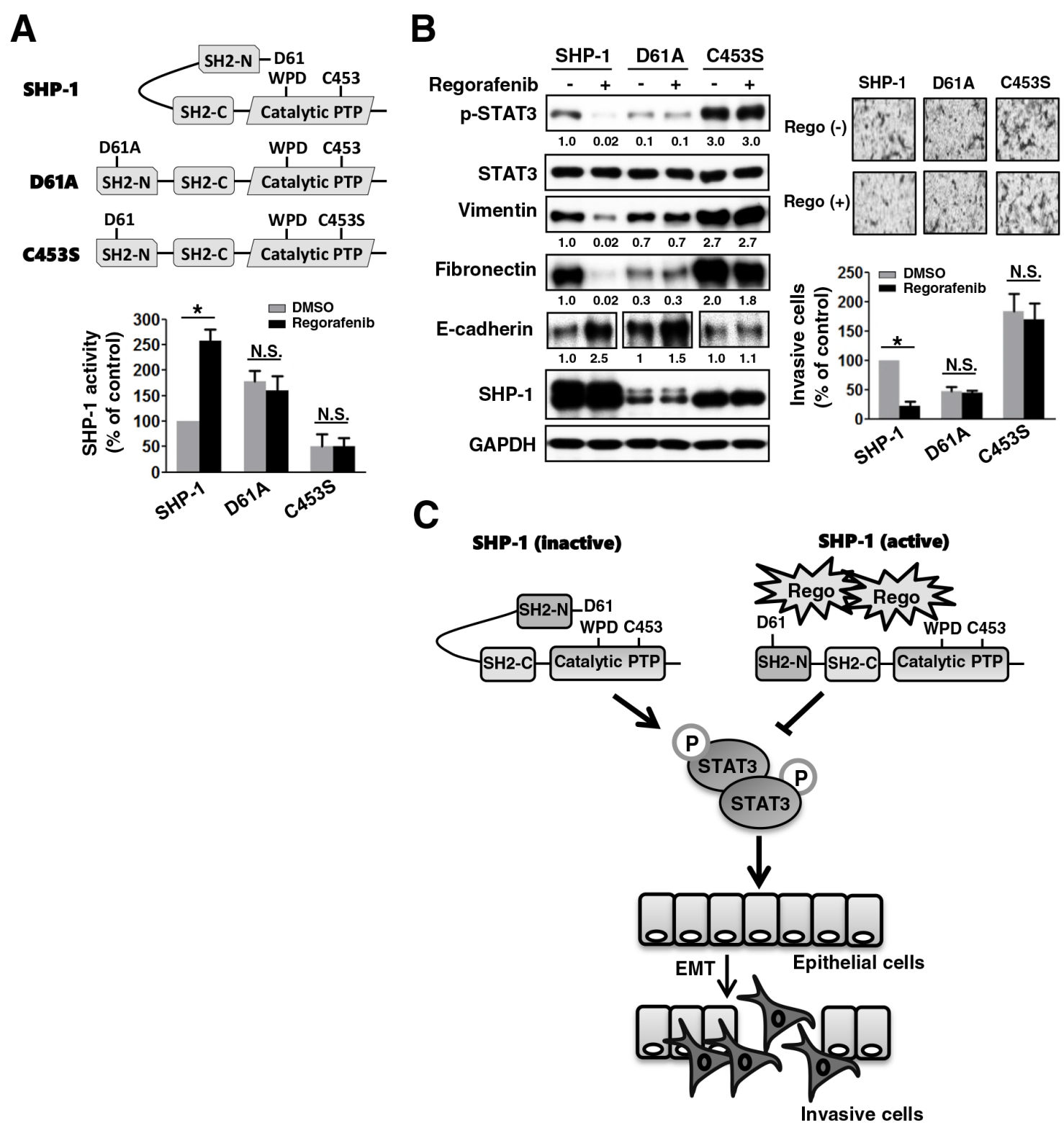

Figure 4: Regorafenib enhances SHP-1 activity through potently relieving the autoinhibition of SHP-1, which reinforces the suppressive effect on p-STAT3Tyr705 and mesenchymal characteristics. A. Upper panels, a schematic representation of wild-type and mutant-type SHP-1 including D61A and C453S carrying constitutive and dead activity of SHP-1, respectively. Lower panels, SHP-1 activity was assessed in the wild-type and mutant-type (D61A and C453S) SHP-1-transfected SW480 cells 2 days after transfection with these plasmids. The results are shown as mean $\pm \mathrm{SD}$ of three independent experiments made in triplicate. (*, $P<0.05$; N.S., non-significant) B. Left panels, western blotting of SHP-1, p-STAT3 ${ }^{\text {Tyr705 }}$, STAT3, and EMT markers in the wild-type and mutant-type (D61 A and C453S) SHP-1-transfected SW480 cells $24 \mathrm{~h}$ after treatment with or without regorafenib at $2.5 \mu \mathrm{M}$. Right panels, the invasive capability was measured in the cells shown in the left panels of (B). The results are shown as mean \pm SD of three independent experiments, made in triplicate. $(*, P<0.05$; N.S., non-significant) C. Regorafenib suppressed TGF- $\beta 1$-induced EMT and invasion in CRC which was mediated through regorafenib-enhanced SHP-1 activity. Regorafenib had the potential to dock to the inhibitory N-SH2 domain and the catalytic PTP domain of SHP-1, resulting in the direct relief of autoinhibition of SHP-1. The activity of SHP-1 tyrosine phosphatase specifically increased the susceptibility to p-STAT3 ${ }^{\text {Tyr705, }}$, which antagonized the EMT pathway. 
relieving the inhibitory N-SH2 domain of SHP-1, leading to reduced $\mathrm{p}-\mathrm{STAT} 3^{\mathrm{Tyr} 705}$ level and eventual suppression of EMT in CRC cells (Figure 4C).

\section{Regorafenib exerts significant anti-metastatic growth of CRC in vivo}

Next, in order to evaluate the therapeutic efficacy of regorafenib on metastasis in vivo, highly metastatic luciferase (Luc) 2-expressing SW480 cells $\left(2 \times 10^{6}\right)$ with mesenchymal characteristics were injected into the tail vein of nude mice and then treated with regorafenib (p.o.,10 mg/kg/day) or vehicle when cells initially showed metastatic growth in the lung monitored by bioluminescent imaging. Importantly, mice receiving regorafenib treatment exhibited a significant reduction in the lung metastatic outgrowth of SW480 cells in comparison with vehicle treatment (Figure 5A and 5B). The data above not only demonstrate that regorafenib is efficacious at curbing metastatic growth of mesenchymal CRC cells in vivo but also further support our in vitro data in suggesting that regorafenib targets SHP-1-dependent STAT3 inactivation during CRC EMT and metastasis.

\section{DISCUSSION}

The cause of the mCRC emergence is highly complex and heterogeneous, and usually results in significant mortality in patients when resistance occurs from the currently available multiple lines of targeted therapies, such as anti-EGFR antibodies cetuximab and panitumumab, and VEGF inhibitors bevacizumab and aflibercept. Recently, regorafenib became the first approved pharmacotherapy for $\mathrm{mCRC}$, serving as a TKI that blocks key molecular markers involved in oncogenesis, angiogenesis and metastasis. Here, we provide further insight into the relationship between the PTPase SHP-1-targeted therapy by regorafenib and CRC EMT reported to be involved in cancer invasion/ metastasis. We provide evidence that regorafenib curbs TGF- $\beta 1$-induced EMT/invasion in vitro by activating

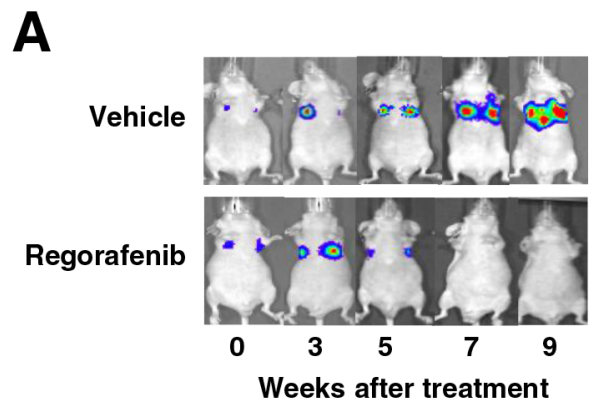

PTPase SHP-1-dependent p-STAT3 ${ }^{\text {Tyr705 }}$ suppression. Increasing PTPase SHP-1 activity by gain-of-function overexpression of wild-type SHP-1 further re-enforces the negative effects of regorafenib on TGF- $\beta 1$-induced p-STAT3 $3^{\text {Tyr705 }}$ and EMT/invasion. Conversely, decreasing PTPase SHP-1 activity significantly restores the negative effects of regorafenib on p-STAT3 ${ }^{\text {Tyr705 }}$ and EMT/invasion as proved by SHP-1 inhibitor or siRNA-mediated SHP1 depletion. Together, we have a novel discovery that regorafenib exerts anti-EMT potency by serving a SHP1 agonist to activate the activity of SHP-1, as an EMTassociated suppressor. Our in vivo data also indicate that regorafenib suppresses metastatic outgrowth of SW480 mesenchymal cells by tail-vein injection metastatic model. However, the sufficient data from in vivo animal study is still lack. Therefore, much more attention should be paid when generating the orthotopic metastatic model that implantation of cells into the spleen and then to see the liver metastasis. Using this methodology in place of examining the lung metastasis by tail-vein injection metastatic model will be a reliable approach to further support our in vitro findings.

Mechanistically, regorafenib effectively increases SHP-1 activity because of the docking potential of regorafenib into the $\mathrm{N}-\mathrm{SH} 2$ and catalytic PTP domain of SHP-1 that relieves the autoinhibiton of SHP-1, resulting in the reduction of both p-STAT3 ${ }^{\text {Tyr705 }}$ and EMT/invasion induced by TGF- $\beta 1$. Interestingly, our previous data indicated that sorafenib, an analog of regorafenib, shows significant anti-EMT activity in vitro and in vivo in a HCC preclinical model via directly activating PTPase SHP-1-dependent p-STAT3 ${ }^{\text {Tyr705 }}$ suppression [14]. Together, these findings firmly confirm that the negative regulatory PTPase SHP-1 is an EMT/ metastasis-suppressing gene, whose activity can be pharmacologically activated by TKIs that act not only as STAT3 inhibitors, but also as SHP-1 agonists, such as regorafenib and sorafenib.

Ample evidence suggests that the loss of E-cadherin is necessary to induce EMT/ metastasis of cancer cells [14-17]. On the other hand, restoration of

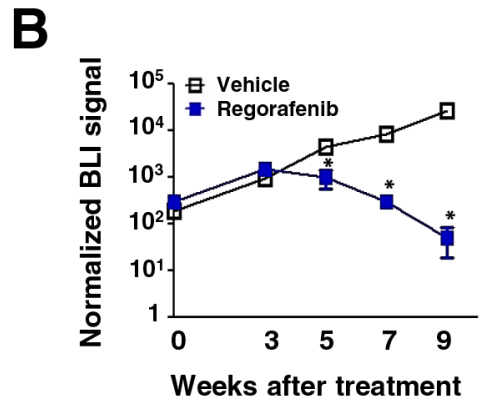

Figure 5: Regorafenib suppresses lung metastatic outgrowth of CRC cells in vivo. A. Representative BLI images of animals in each experimental group at the indicated time points after receiving the indicated drug treatment. B. Normalized BLI signals of lung metastatic outgrowth in mice $(\mathrm{n}=5)$ receiving regorafenib $(10 \mathrm{mg} / \mathrm{kg} /$ day $)$ or vehicle, daily, when metastatic growth was initially seen in the lung after tail vein injection with Luc2-expressing SW480 cells $\left(2 \times 10^{6}\right)$. Points, mean $(\mathrm{n}=5)$; bars, SEM. *, $P<0.05$. 
E-cadherin expression enhances intercellular adhesion, and suppresses the EMT/metastasis [14, 21, 22]. Here, through assay of 243 CRC patients by a Kaplan-Meier analysis, the median OS was not available in patients with strong expression both in SHP-1 and E-cadherin. In contrast, patients with weak expression of both SHP-1 and E-cadherin had a more significant reduction in median OS than others. These observations imply that patients with highly levels of SHP-1-E-cadherin expression display longer survival. In addition, the clinical data reveal that a positive correlation significantly existed between SHP-1 and E-cadherin expression in $\mathrm{CRC}$ tissues. Interestingly, the expression of E-cadherin, but not SHP-1, had an inverse correlation with histological grading. These data suggest that SHP1 may not be the only upstream positive regulator of E-cadherin. Other upstream regulators may also positively control the expression of E-cadherin to finally establish the adherens junctions. For example, GSK-3 $\beta$ positively regulates E-cadherin by negatively controlling the stability of SNAIL/SLUG, as the repressors of E-cadherin [23]. In addition, MicroRNA-200a has been reported to function as an EMT-associated tumor suppressor to upregulate E-cadherin by downregulating ZEB1/2, as the repressors of E-cadherin [24]. Therefore, E-cadherin, but not SHP-1, negatively correlates with histological grading may due to the reason that other upstream regulators of E-cadherin are essential to induce the expression of E-cadherin, which serves as a direct downstream effector and significantly associates with the low-grading cells with well differentiation. SHP-1 is one of the upstream positive regulators of E-cadherin and not an independent effector to directly associate with histological grading in the present study. Collectively, these clinical data are further confirming the in vitro findings that SHP-1 is an EMT-associated suppressor to upregulate E-cadherin by suppressing p-STAT3 ${ }^{\text {Tyr705. }}$.

In summary, this is the first report showing a novel link between regorafenib and CRC EMT. Regorafenib guides anti-EMT/metastasis therapy in vitro and in vivo, through the activation of PTPase SHP-1-dependent

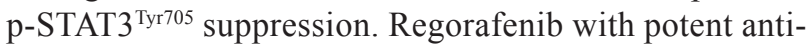
EMT activity serves as a SHP-1 agonist by the relief of autoinhibition in SHP-1. Of note, SHP-1 is identified as not only as a suppressor of EMT/metastasis but also as a pharmacologic target of regorafenib. Further, we establish a novel link between the SHP-1-E-cadherin axis and overall survival of CRC pateints. The findings of this study may guide the development of alternative PTPase-targeted therapy for $\mathrm{mCRC}$ patients who have failed multiple lines of therapy, and shows that antiEMT potency in regorafenib significantly activates PTPase SHP-1-dependent STAT3 inactivation, and might provide a straightforward pharmacotherapy for $\mathrm{mCRC}$.

\section{MATERIALS AND METHODS}

\section{Cell culture}

CRC cell lines, including Hct-116, HT-29 and SW480 cell lines were maintained in RPMI1640 medium supplemented with $10 \% \mathrm{FBS}, 100$ units $/ \mathrm{ml}$ of penicillin and streptomycin (Invitrogen, Carlsbad, CA, USA), and then incubated at $37^{\circ} \mathrm{C}$ in a humidified $5 \% \mathrm{CO}_{2}$ atmosphere.

\section{Reagents and plasmids}

Recombinant human TGF- $\beta 1$ was purchased from R\&D Systems (Minneapolis, MN). Regorafenib was kindly provided by Bayer HealthCare Pharmaceuticals. For in vitro studies, regorafenib was dissolved in dimethyl sulfoxide and then added to the cells maintained in RPMI 1640 medium without FBS. For in vivo animal study, regorafenib was suspended in Kolliphor (JT Baker). SHP1 inhibitor (PTP III) was purchased from Calbiochem. Smart-pool siRNA, including control (D-001810-10), SHP-1 (PTPN6, L-009778-00-0005) were all purchased from Dharmacon (Chicago, IL). Plasmids of human wild-type STAT3 and SHP-1 (PTPN6) were encoded by pCMV6 vector with myc-tag. For mutant-type SHP-1 expression, we generated a D61A plasmid that changed aspartic acid at 61 to an alanine residue, which was cloned into pCMV6-Entry vector. Mutant-type SHP-1 C453S was encoded by pJ3-SHP-1 plasmid purchased from Addgene plasmid repository (http://www.addgene.org/). Smart-pool siRNA, including control (D-001810-10), SHP-1 (PTPN6, L-009778-00-0005) were purchased from Dharmacon (Chicago, IL). These plasmids or siRNA were subsequently transfected into cells by using Lipofectamine 2000 reagent (Invitrogene, CA).

\section{SHP-1 phosphatase activity}

The cellular protein extracts were incubated with anti-SHP-1 antibody in immunoprecipitation buffer overnight. Protein G-Sepharose 4 Fast flow (GE Healthcare Bio-Science, NJ) was added to each sample, followed by incubation for $3 \mathrm{~h}$ at $4{ }^{\circ} \mathrm{C}$ with rotation. A RediPlate 96 EnzChekR Tyrosine Phosphatase Assay Kit (R-22067) was used for SHP-1 activity assay (Molecular Probes, Invitrogen, CA).

\section{In vitro invasion assay}

The invasive capability of cells was examined using polycarbonate transwell filters containing $8-\mu \mathrm{m}$ pores (Corning Coster, Cambridge, MA). Cells $\left(3 \times 10^{5}\right)$ seeded in serum-free medium on the upper side of the chamber coated with Matrigel (BD Biosciences, Bedford, MA, USA). The cells were allowed to migrate toward the lower 
chamber containing media supplemented with 10\% FBS. After 24-hours, cells on the lower side of the membrane were fixed, stained with crystal violent and then counted. The average number of the crystal violent stained-cells was calculated by three independent experiments. The data of invasive cells is presented as a percentage normalized to the control according to each experiment.

\section{Immunofluorescent staining}

Cells seeded on a coverslip were fixed in $4 \%$ paraformaldehyde, permeabilized with $0.25 \%$ Triton $\mathrm{X}-100$, and then blocked with $5 \%$ bovine serum albumin (BSA). After that, primary antibodies were incubated against vimentin (Abcam), E-cadherin (Abcam). Rhodamine phalloidin (Invitrogen) and DAPI were used for F-actin and nuclear staining, respectively. The average intensity of vimentin and E-cadherin was determined by densitometry Image J software. Statistical analyses were performed using SPSS 13.0 for Windows (SPSS Inc., Chicago, IL, USA).

\section{Western blotting}

Whole-cell lysates were made in RIPA buffer and subjected to SDS-PAGE, transferred onto a polyvinylidene difluoride membrane (Millipore, Billerica, MA, USA) and incubated with primary antibody, and then incubated with horseradish peroxidase-conjugated secondary antibodies. Specific proteins were detected using enhanced chemiluminescence reagent. The primary antibodies

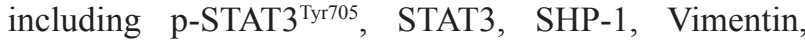
Fibronectin, E-cadherin and GAPDH were purchased from Abcam (Cambridge, MA). For quantification of protein levels on chemiluminescent western blots, densitometry Image J software was employed. Statistical analyses were performed using SPSS 13.0 for Windows (SPSS Inc., Chicago, IL, USA).

\section{In vivo animal study}

Luciferase $($ Luc $)$ 2-expressing SW480 cells $\left(2 \times 10^{6}\right)$ suspended in $100 \mu \mathrm{L}$ PBS were injected into the tail vein of 6-week-old nude mice. Mice were randomly grouped into regorafenib- (p.o., $10 \mathrm{mg} / \mathrm{kg} /$ day) or a vehicletreatment group when cells were initially observed to metastasize to the lung and monitored by bioluminescent imaging (BLI). To evaluate the therapeutic efficacy of regorafenib on the metastatic outgrowth of SW480 cells in lungs, BLI was used for regorafenib- or vehicle-treated mice on weeks $0,3,5,7$, and 9 .

\section{Patient specimens}

Patients with colorectal cancer, determined according to the World Health Organization criteria, were enrolled from January 2002 through January 2014 and classified according to the American Joint Committee on Cancer (AJCC) staging system (Version 6). Clinical data were obtained from the cancer registry. Overall survival (OS) was defined as the time from primary resection to death from cancer. The follow-up period ended in January 2014 , or at the time of death of the patient. Left colon cancer was defined as a malignancy in the splenic flexure, descending colon, sigmoid colon, rectosigmoid colon and rectum.

\section{Immunohistochemistry}

For tissue microarray (TMA) and immunohistochemistry (IHC), the procedures followed our previous methods and the manufacturer's instructions [20]. SHP-1 and E-cadherin antibodies were purchased from Abcam (Cambridge, MA). Omission of the primary antibody served as a negative control. Immunopositive results were evaluated by 2 pathologists. The intensity of stained cells was scored as $0,1,2$, or 3 . Percentages of stained cells were counted. A final immunohistochemical score (H-score) was calculated by summing the products of the staining intensities $(0-3)$ and distributions $(0 \%-$ $100 \%$ ). H-scores ranged from 0 to 300 . An H-score equal to or greater than 150 was defined as strongly positive for SHP-1 staining; all others were scored as weakly positive. An H-score greater than 150 was defined as strongly positive for E-cadherin staining; all others were scored as weakly positive.

\section{Statistical and survival analysis}

Quantitative data are presented as the mean \pm standard deviation (SD) from three independent experiments. The $t$ test was used to compare age distribution. The correlations between clinicopathological variables and immunopositivity were analyzed using the $\chi^{2}$ test or Fisher's exact test. Survival was estimated using the Kaplan-Meier method. A 2-sided P value of less than 0.05 was regarded as statistically significant. SPSS software (version 16.00, SPSS, Chicago, IL) was used for all the statistical analyses.

\section{ACKNOWLEDGMENTS}

This work was supported by grants MOST 105-2314-B-002-190-MY2, MOST 103-2622-B-002-006, MOST 103-2325-B-002-016, and 104C101-62 from the Ministry of Science and Technology, Taiwan and College of Medicine, National Taiwan University.

\section{CONFLICTS OF INTEREST}

We have no potential conflicts of interest to disclose. 


\section{GRANT SUPPORT}

As the description in Acknowledgements.

\section{REFERENCES}

1. Grothey A, Van Cutsem E, Sobrero A, Siena S, Falcone A, Ychou M, Humblet Y, Bouché O, Mineur L, Barone C, Adenis A, Tabernero J, Yoshino T, et al. Regorafenib monotherapy for previously treated metastatic colorectal cancer (CORRECT): an international, multicentre, randomised, placebo-controlled, phase 3 trial. Lancet. 2013; 381:303-312.

2. Wilhelm SM, Dumas J, Adnane L, Lynch M, Carter CA, Schütz G, Thierauch KH, Zopf D. Regorafenib (BAY 73-4506): a new oral multikinase inhibitor of angiogenic, stromal and oncogenic receptor tyrosine kinases with potent preclinical antitumor activity. Int $\mathrm{J}$ Cancer. 2011; 129:245-255.

3. Abou-Elkacem L, Arns S, Brix G, Gremse F, Zopf D, Kiessling F, Lederle W. Regorafenib inhibits growth, angiogenesis, and metastasis in a highly aggressive, orthotopic colon cancer model. Mol Cancer Ther. 2013; 12:1322-1331.

4. Qin Y, Tang B, Hu CJ, Xiao YF, Xie R, Yong X, Wu YY, Dong H, Yang SM. An hTERT/ZEB1 complex directly regulates E-cadherin to promote epithelial-to-mesenchymal transition (EMT) in colorectal cancer. Oncotarget. 2016; 7:351-361. doi: 10.18632/oncotarget.5968.

5. Chen HN, Yuan K, Xie N, Wang K, Huang Z, Chen Y, Dou Q, Wu M, Nice EC, Zhou ZG, Huang C. PDLIM1 stabilizes the E-cadherin/beta-catenin complex to prevent epithelial-mesenchymal transition and metastatic potential of colorectal cancer cells. Cancer Res. 2016; 76:1122-1134.

6. Zhang J, Zhu L, Fang J, Ge Z, Li X. LRG1 modulates epithelial-mesenchymal transition and angiogenesis in colorectal cancer via HIF-1alpha activation. J Exp Clin Cancer Res. 2016; 35:29.

7. Zhang F, Luo Y, Shao Z, Xu L, Liu X, Niu Y, Shi J, Sun X, Liu Y, Ding Y, Zhao L. MicroRNA-187, a downstream effector of TGFbeta pathway, suppresses Smad-mediated epithelial-mesenchymal transition in colorectal cancer. Cancer Lett. 2016; 373: 203-213.

8. Yi TL, Cleveland JL, Ihle JN. Protein tyrosine phosphatase containing SH2 domains: characterization, preferential expression in hematopoietic cells, and localization to human chromosome 12p12-p13. Mol Cell Biol. 1992; 12: 836-846.

9. Adachi M, Fischer EH, Ihle J, Imai K, Jirik F, Neel B, Pawson T, Shen S, Thomas M, Ullrich A, Zhao Z. Mammalian SH2-containing protein tyrosine phosphatases. Cell. 1996; 85:15.

10. Tai WT, Cheng AL, Shiau CW, Huang HP, Huang JW, Chen PJ, Chen KF. Signal transducer and activator of transcription 3 is a major kinase-independent target of sorafenib in hepatocellular carcinoma. J Hepatol. 2011; 55:1041-1048.

11. Liu CY, Tseng LM, Su JC, Chang KC, Chu PY, Tai WT, Shiau CW, Chen KF. Novel sorafenib analogues induce apoptosis through SHP-1 dependent STAT3 inactivation in human breast cancer cells. Breast Cancer Res. 2013; 15: R63.

12. Fan LC, Teng HW, Shiau CW, Tai WT, Hung MH, Yang SH, Jiang JK, Chen KF. Pharmacological Targeting SHP1-STAT3 Signaling Is a Promising Therapeutic Approach for the Treatment of Colorectal Cancer. Neoplasia. 2015; 17: 687-696.

13. Fan LC, Teng HW, Shiau CW, Lin H, Hung MH, Chen YL, Huang JW, Tai WT, Yu HC, Chen KF. SHP-1 is a target of regorafenib in colorectal cancer. Oncotarget. 2014; 5:6243-6251. doi: 10.18632/oncotarget.2191.

14. Fan LC, Shiau CW, Tai WT, Hung MH, Chu PY, Hsieh FS, Lin H, Yu HC, Chen KF. SHP-1 is a negative regulator of epithelial-mesenchymal transition in hepatocellular carcinoma. Oncogene. 2015; 34:5252-5263.

15. Luo F, Xu Y, Ling M, Zhao Y, Xu W, Liang X, Jiang R, Wang B, Bian Q, Liu Q. Arsenite evokes IL-6 secretion, autocrine regulation of STAT3 signaling, and miR-21 expression, processes involved in the EMT and malignant transformation of human bronchial epithelial cells. Toxicol Appl Pharmacol. 2013; 273: 27-34.

16. Rokavec M, Oner MG, Li H, Jackstadt R, Jiang L, Lodygin D, Kaller M, Horst D, Ziegler PK, Schwitalla S, SlottaHuspenina J, Bader FG, Greten FR, et al. IL-6R/STAT3/ miR-34a feedback loop promotes EMT-mediated colorectal cancer invasion and metastasis. J Clin Invest. 2014; 124:1853-1867.

17. Xiong H, Hong J, Du W, Lin YW, Ren LL, Wang YC, Su WY, Wang JL, Cui Y, Wang ZH, Fang JY. Roles of STAT3 and ZEB1 proteins in E-cadherin down-regulation and human colorectal cancer epithelial-mesenchymal transition. J Biol Chem. 2012; 287:5819-5832.

18. Zhang Z, Bai Y, Li P, Zhao J, Wang Y, Sun L, Tang J. Relationship between activated STAT3 protein and epithelial-mesenchymal transition in papillary thyroid carcinoma. [Article in Chinese]. Lin Chung Er Bi Yan Hou Tou Jing Wai Ke Za Zhi. 2013; 27: 1265-1268.

19. Tai WT, Shiau CW, Chen PJ, Chu PY, Huang HP, Liu CY, Huang JW, Chen KF. Discovery of novel Src homology region 2 domain-containing phosphatase 1 agonists from sorafenib for the treatment of hepatocellular carcinoma. Hepatology. 2014; 59:190-201.

20. Teng HW, Yang SH, Lin JK, Chen WS, Lin TC, Jiang JK, Yen CC, Li AF, Chen PC, Lan YT, Lin CC, Hsu YN, Wang $\mathrm{HW}$, et al. CIP2A is a predictor of poor prognosis in colon cancer. J Gastrointest Surg. 2012; 16:1037-1047.

21. Perl AK, Wilgenbus P, Dahl U, Semb H, Christofori G. A causal role for E-cadherin in the transition from adenoma to carcinoma. Nature. 1998; 392:190-193. 
22. Cano A, Pérez-Moreno MA, Rodrigo I, Locascio A, Blanco MJ, del Barrio MG, Portillo F, Nieto MA. The transcription factor snail controls epithelial-mesenchymal transitions by repressing E-cadherin expression. Nat Cell Biol. 2000; 2:76-83.

23. Medici D, Hay ED, Olsen BR. Snail and Slug promote epithelial-mesenchymal transition through
beta-catenin-T-cell factor-4-dependent expression of transforming growth factor-beta3. Mol Biol Cell. 2008; 19:4875-4887.

24. Su J, Zhang A, Shi Z, Ma F, Pu P, Wang T, Zhang J, Kang C, Zhang Q.MicroRNA-200a suppresses the Wnt/ $\beta$-catenin signaling pathway by interacting with $\beta$-catenin. Int $\mathrm{J}$ Oncol. 2012; 40:1162-1170. 